Tipon, salin, at suri ng mga kuwentong bayang Iloko

Galdonez, Dominic Patric $\bowtie$

Philippine Science High School-Ilocos Region Campus, Philippines (galdonezdg@irc.pshs.edu.ph)

Received: 5 November 2021 Available Online: 2 January 2022
Revised: 29 November 2021 DOI: $10.5861 /$ ijrse. 2022.205

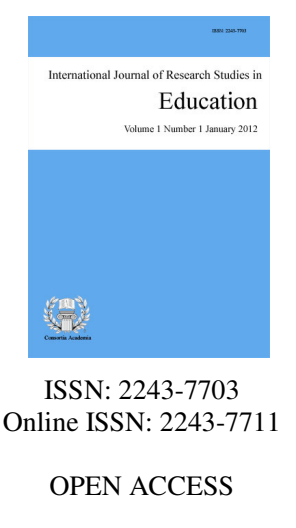

\title{
Abstract
}

This study is generally designed to collect, translate, and analyze Ilocano folk stories. Specifically, it sought to answer the following objectives: determine the approaches and characteristics used and discovered in translating the texts; and know the different Ilocano cultures entrenched in the stories. This qualitative research collected fifteen (15) folk stories written in Ilocano, translated these in Filipino, and validated by the experts. Using the concepts of Newmark, translated folk stories were analyzed to determine the approaches and characteristics used in the translation. Furthermore, originally written folk stories in Ilocano were analyzed to establish the themes on culture. Based on data, the following approaches were used by the translator: transposition, naturalization, adding, gisting, functional translation, couplet, and lifting of terms from the source language. All translated folk stories have the following characteristics: literal, free, culture-based, and appropriate. Four themes were formulated to explain the Ilocano culture embedded in the folk stories: Labor-Culture, Family Culture, Positive Traits, and Negative Traits. The implications of the study are the concepts of Newmark in translation are essential in translating texts especially prose; lifting of terms from the source language could be a strategy to enrich the intelectuallization of the language; translating literature or texts must be appropriate and contextualized; and Ilocano folk stories are rich in values and culture.

Keywords: translation, folk stories, culture, Ilocano, Newman 


\section{Tipon, salin, at suri ng mga kuwentong bayang Iloko}

\section{Introduksiyon}

Sa patuloy na pagyabong ng makabagong teknolohiya at iba pang mga salik, marami pa ring mga bagay-bagay ang patuloy na inaapuhap, inaaral, at sinusuri. Karamihan sa mga ito ay nasa paligid lang natin ngunit may kakulangan ang nakararami sa pagpansin sa mga ito. Isa sa mga maituturing na kailanma'y di naglalahong pamana ng ating mga ninuno sa kabila ng mga salik na nabanggit ay ang mga pasulat at pasalitang piyesang pampanitikan. Ayon kay Du (2014), ang panitikan ay isang bukas na talaarawan ng mga mamamayan. Sa talaarawang ito, mababasa ang panahon ng kasaysayan at kultura ng isang pangkat. Ibig sabihin, nakaangkla na talaga ang karanasan, nagagad, at naisadokumento na halos ang pang-araw-araw na pamumuhay ng tao dahil sa panitikan. Sa kabila ng kaalamang ito, marami pa ring mga katutubong mga panitikan ang patuloy na nakakubli sa mata ng nakararami. Nangyayari ang penomenong ito dahil sa hindi lubos na pagtangkilik ng marami sa mga kagawiang ito. Ayon kay Lumbera (2008), sa pagbanggit ni Santos (2008), maraming mga salik ang nakaaapekto sa pagsulong ng literaturang Filipino. Kabilang rito ang outlet, edukasyon, ekonomiya, at pulitika. Isang malaking problema ng panitikang Filipino ang outlet para sa mga akdang nasa wikang Filipino.

Mayaman ang Pilipinas sa mga akdang pampanitikan na binubuo ng iba't ibang anyo gaya na lamang ng mga alamat, kuwentong bayan, epiko, tula, at iba pa. Ayon kay Ramos (1984) sa pagbanggit ni Catipay (2019), ang panitikang Filipino ay may pambihirang kabuoan. Ito'y nagsisimula sa mga pasalitang tradisyon- tulad ng mga kuwentong bayan. Ang mga ito ay isa sa mga mahahalagang mukha ng panitikan sapagkat ito ang bumubuo ng identidad ng isang lipunan. Ang mga panitikang ito ay koleksiyon ng mga kuwentong nag-uugnay sa mga tao, lugar, hayop, at mga bagay na nasa loob mismo sa konteksto ng Pilipinas. Ang mga ito ay ekspresyon ng kultura na purong Pilipino na galing sa mayamang nakaraan hanggang sa pag-usbong nito sa kasalukuyan hanggang sa hinaharap, kasaganaan, pagkakaisa, at kooperasyon (Young, 1994). Kaya't marami sa mga Pilipino ang nag-apuhap sa mga genreng pampanitikang ito upang pag-aralan, lalong-lalo na sa kaso ng mga katutubong panitikan. Ang ilan sa mga pag-aaral na ito ay ang pagsusuri sa nilalaman ng mga akda na sumasalamin sa pang-araw-araw na pamumuhay ng mga Pilipino at ang pagtupad sa mga hangarin sa buhay. Ang pagtukoy sa mga kultural na konteksto o pagpapahalaga sa mga akdang pampanitikan ay nakatutulong upang payabungin ang mga magagandang asal at tuluyang tanggalin ang mga di kaaya-ayang mga pag-uugali. Ang pagtitipon at pagsusuri sa mga akdang pampanitikan ay mga hakbang upang mapayabong ang kultura at panitikan ng isang bansa.

Maliban sa pagtitipon at pagsusuri, mahalaga rin ang pagsasalin sa preserbasyon at pagpapayabong ng kultura, tradisyon, at lalong-lalo na ng panitikan ng isang bansa. Ang pagsasalin ay isa sa mga isinusulong na pamamaraan at hakbang ng Komisyon sa Wikang Filipino upang mapayabong ang kasalukuyang leksikon ng wikang Filipino. Nagsisilbi rin itong isang malaking pundasyon para sa intelektuwalisasyon ng pambansang wika. Ayon kay Almario (2015), dapat mapansin ang mahigpit na ugnayan ng pagsasalin at pagpapaunlad ng Wikang Pambansa upang makamit ang antas ng kultibasyon (modernisasyon o intelektuwalisasyon) na inaasam-asam at upang makamit ang titulo ng wika ng karunungan.

Buhat sa mga nabasa at nasuring mga konsepto, nilayon ng mananaliksik na tipunin, isalin, at suriin ang ilang piling kuwentong bayang nakasulat sa wikang Ilokano. Ang mga natipong akda ay hindi pa naisasalin at kakikitaan nang mahusay na pagkakagawa at pagkakasulat na maaaring gamitin bilang kagamitang panturo. Ang pag-aaral na ito ay malaking tulong upang mapayabong ang kaalaman ng mga tao sa lipunan sa mga akdang pampanitikan at para na rin sa pagpapayabong ng wika at panitikang pambansa.

Layunin ng Pag-aaral - Ang pag-aaral na ito ay naglayong magtipon, magsalin, at magsuri ng mga kuwentong bayan ng mga Ilokano. Tiniyak ding masuri ang mga angkop na pamamaraang nagamit sa pagsasalin;

38 Consortia Academia Publishing (A partner of Network of Professional Researchers and Educators) 
matukoy ang katangian ng mga naisaling akda batay sa mga eksperto; at madiskubre ang mga kalinangang napapaloob sa mga kuwentong bayang Iloko.

\section{Metodolohiya}

Sa bahaging ito, makikita ang disenyo, instrumento, at pamamaraan sa pangangalap at pagsusuri sa mga datos ng pag-aaral.

\subsection{Disenyo ng Pag-aaral}

Ang pag-aaral na ito ay gumamit ng kuwalitatibong lapit ng pananaliksik. Sinuri ang mga nakalap na datos gamit ang mga disenyong conventional at summative content analysis. Ang conventional content analysis ay isang disenyong pampananaliksik na naglalayong mailarawan ang isang penomenon lalong-lalo na kung may limitado at/o kakaunting pag-aaral tungkol dito. Ang mga mananaliksik ay hindi kinakailangang maghaka ng mga bagay-bagay o kategorya tungkol sa penomenon (Kondracki at Wellman, 2002). Ginamit ang disenyong ito sa pagsusuri sa mga nakalapat na mga kultural na pananaw sa mga kuwentong bayang isinalin at sinuri. Sa punto naman ng summative content analysis, kinakailangan ang disenyong ito sa pagsasalin ng mga datos na nakalap sa pag-aaral. Sa paggamit ng disenyong ito, ayon kina Potter at Levine-Donnerstein (1999) na sinipi nina Hsiu-Fang (2005) ay kinakailangan tukuyin at tayahin ang mga salita at/o nilalaman upang maintindihan ang mga ito sa paraang kontekstuwal. Ang disenyong ito ay ginamit upang isalin ang mga kuwentong bayan na nakasulat sa wikang Ilokano tungo sa wikang Filipino.

\subsection{Instrumento at Kasangkot sa Pag-aaral}

Sa pag-aaral na ito, binigyang pansin ang mga kuwentong bayan ng mga Ilokano. Natukoy at natipon ang labinlimang (15) kuwentong bayang nasusulat sa Ilokano. Ang mga ito ay ang sumusunod: Anghel ni Guillermo R. Andaya; Komusta, Apo Abogado? ni Estanislao Tenoso; Apong Ciano: Taga-Lubong ni Reynaldo A. Duque; Arbis ti Sardam ni Estrella C. Miranda; Pusa ni Benjamin M. Pascual; Bunga ni Hermogenes F. Belen; Dua a Pisos ni Cresecencio S. Barayuga; Agluom Manen dagiti Lansones ni T. Gabriel Tugade; Sumangpeten ni Tatang ni Consorcio Borje; Mision Numero 9 ni Mariano U Foronda; Kanta iti amin a Panawen ni Elpidio Rimando; Agkitemda iti Malem ni Angel C. Anden; Nalabaga ti Pasayak ni Herminio C. Calica; at Dagiti Alimatek ni Cecilio V. Gasmen. Natukoy at natipon ang mga kuwentong bayan sa pamamagitan ng document analysis at pagsasaalang-alang sa inihandang inklusyon at eksklusyong pamantayan. Ginamit ang purposive sampling para tukuyin ang mga ekspertong tumasa at tumaya sa mga salin. Ang mga tumasa at tumaya ng isinagawang salin ay tatlong guro/propesor na nagtuturo ng pagsasalin at may karanasan na sa pagsasalin ng akdang pampanitikan mula sa wikang Ilokano tungo sa wikang Filipino. Nakapaglathala na rin sila ng mga akdang pampanitikang may tuon sa pagsasalin. Ipinataya rin ang mga salita o katawagang kultural sa mga eksperto sa wikang Ilokano para higit na maayos at makinis ang mga isinagawang pagsasalin sa mga akda.

\subsection{Pagsusuring Datos}

Sa pag-aaral na ito, nilikom at pinili ang labinlimang (15) kuwentong bayan ng mga Ilokano. Gumamit ang mananaliksik ng document analysis para mataya ang mga ginamit na mga kuwentong bayan na pinaghanguan ng datos sa isinagawang pananaliksik. Napili ang mga natukoy na kuwentong bayan dahil orihinal na nakasulat ang mga ito sa wikang Ilokano para matamo ang mithiing pagsasalin. Ang mga kuwentong bayang napili ay isinulat, inilathala, at tinipon ng ilang mga kilalang manunulat na taal sa wika at kalinangang Iloko na lalong nagpaigting para piliin ang mga ito. Natukoy din na ang mga kuwentong bayan ay kailanma'y hindi pa naisasalin sa anomang wika lalong-lalo na sa wikang Filipino. Napansin din sa naging pagsusuri ng mga kuwentong bayang pinili na may mga kalinangang Ilokano na napapaloob sa mga ito. Isinalin ng mananaliksik ang lahat ng mga kuwentong bayang natipon. Matapos maisalin ang mga nasabing kuwentong bayan, ipinatasa at ipinataya ang kaangkupan ng mga salin sa mga natukoy na tagataya/eksperto. Batay sa awtput at mga komento ng pagtatasa at pagtataya ng 
Galdonez, D. P.

mga eksperto, natukoy ang mga ginamit na paraan sa pagsasalin. Ito ay naibatay sa mga konsepto ni Newmark (1988) sa pagsasalin. Tiningnan din ang katangian ng mga isinaling akda sa pamamagitan ng pagsusuri sa isinagawang pagtataya, komento, at mungkahi ng mga eksperto. Ginamit din ang cool and warm na pagsusuri upang matukoy ang mga tema na sumasalamin sa kultura at pamumuhay ng mga Ilokano at ang mga penomenon sa isinagawang pagsasalin.

\section{Mga Resulta at Pagtatalakay}

Makikita sa bahaging ito ang mga resulta at pagpapakahulugan sa mga datos na nakalap at nasuri sa pag-aaral.

\subsection{Natuklasang Angkop na Pamamaraan sa Pagsasalin}

\section{Talahanayan 1}

Natuklasang Angkop na Pamamaraan sa Pagsasalin

\begin{tabular}{|c|c|}
\hline Pamamaraan & Pahayag mula sa mga akda \\
\hline \multirow[t]{3}{*}{ Transposisyon } & Ulan sa Takipsilim \\
\hline & Ilokano (SL): Timmakder ni Baket Marcela. \\
\hline & Filipino (TL): Si Baket Marcela ay tumayo. \\
\hline \multirow[t]{6}{*}{ Naturalisasyon } & Bunga \\
\hline & Ilokano (SL): "Maysa laeng dayta a pagtaktakan ti \\
\hline & panawen," kinuna ti panglakayen ti barrio idi \\
\hline & sinarungkaran ni Marcelo. \\
\hline & Filipino (TL): "Isa lang iyan sa mga nag-uubos ng \\
\hline & $\begin{array}{l}\text { panahon," sinabi ng panglakayen ng baryo nang binisita ito } \\
\text { ni Marcelo. }\end{array}$ \\
\hline \multirow[t]{3}{*}{ Pagdaragdag } & Agluom Manen dagiti Lansones ni T. Gabriel Tugade \\
\hline & Ilokano (SL): Nagmataak. \\
\hline & Filipino (TL): Binuksan ko ang aking mga mata. \\
\hline \multirow{5}{*}{ Pagkakaltas } & Agkitemda Iti Malem \\
\hline & Ilokano (SL): Agsikig komat bassit ngem narigat met la \\
\hline & unayen ti panagkutina. \\
\hline & Filipino (TL): Tatagilid na lang ito ngunit nahihirapan pa \\
\hline & sa paggalaw. \\
\hline \multirow[t]{2}{*}{ Functional na pagtutumbas } & Nalabaga ti pasayak \\
\hline & $\begin{array}{l}\text { Ilokano (SL): Insurot ni Andres ti panagkitana iti narakab a } \\
\text { bukot ni Victor ken ti makapungtot a widawidna a } \\
\text { dinanggayan ti panaglitaklitak ti sikakaluban a talunasanna. } \\
\text { Filipino (TL): Ipinukol ni Andres ang kaniyang tingin sa } \\
\text { payat na batok ni Victor at ang nagagalit na galaw nito na } \\
\text { sinabayan ng huni ng natakpang itak nito. }\end{array}$ \\
\hline \multirow[t]{3}{*}{ Kuplet } & Misyon Bilang 9 \\
\hline & $\begin{array}{l}\text { Ilokano (SL): Inirutan ni Dr. Venancio Ronquillo ti ruskas } \\
\text { iti batog ti rusokna. Tinaliawna dagiti innem a kaduana a } \\
\text { nakasimpan iti tugawda. }\end{array}$ \\
\hline & $\begin{array}{l}\text { Filipino (TL): Hinigpitan ni Dr. Venancio Ronquillo ang } \\
\text { turnilyo sa kaniyang tiyan. Nilingon niya ang anim na } \\
\text { kasamahan na nakaupo. }\end{array}$ \\
\hline \multirow{4}{*}{$\begin{array}{l}\text { Pag-aangat sa/ng orihinal } \\
\text { na wika (dagdag mula sa mananaliksik) }\end{array}$} & Dagiti Alimatek \\
\hline & Ilokano (SL) : Idi makagteng iti nagpikoran ti kalsada \\
\hline & $\begin{array}{l}\text { mayor, kinamangna ti bit-ang nga agpaabagatan iti likudan } \\
\text { ti kombento. }\end{array}$ \\
\hline & $\begin{array}{l}\text { Filipino (TL): Nang makarating ito sa lilikuhan ng kalsada } \\
\text { mayor, tinahak niya ang bit-ang na darakong timog sa may } \\
\text { likuran ng kumbento. }\end{array}$ \\
\hline
\end{tabular}

Note: Simulaang Lengguwahe (SL); Target na Lengguwahe (TL)

Transposisyon - Sa isinagawang pagsasalin, isa sa pamamaraang nagamit ay ang transposisyon Ito ay isang pamamaraan ng pagsasalin kung saan nagpapalit ang posisyon ang simuno at panaguri o ang pagpapalit ng lugar ng mga salita sa loob ng pangungusap. Ayon kay Whittlesey (2012) sa ganitong pamamaraan may pagtatangkang mapalitaw ang konteksto ng orihinal na akda sa pamamagitan ng pagpapalit-posisyon ng mga salita dahil sa 
Tipon, salin, at suri ng mga kuwentong bayang Iloko

sosyo-historikal na katangian ng wikang pinagsasalinan ngunit napapanatili ang kahulugan ng isinalin. Sa pangungusap, sa bahagi ng simulaang lengguwahe (SL), makikita ang pandiwa bilang panaguri sa pangungusap na nauuna kaysa sa paksang pinag-uusapan, samantalang sa target na lengguwahe (TL) naging karaniwan ang ayos ng pangungusap. Nauuna ang paksang pinag-uusapan kaysa sa pandiwa na ginamitan pa ng pangawing na "ay" na walang tumbas sa SL. Mapapansin sa unang pangungusap ang pag-iiba ng posisyon ng mga salita sa pangungusap at ang pagdaragdag ng pangawing na "ay" ngunit walang nakitang pag-iiba sa kahulugan ng pahayag. Napansin din ang ganitong pamamaraan ng pagsasalin sa naging pag-aaral at pagsusuri ni Jamilosa-Silapan (1993) sa naging pagsasalin ni Jose A. Bragado sa kuwentong Buneng kung saan inilipat niya ang posisyon ng ilang salita sa kaniyang pagsasalin ngunit hindi ito naging hadlang sa pagpapakinis ng isinagawang pagsasalin.

Naturalisayon - Ito ay adapsyon ng salita mula sa SL na sinusunod ang istandard na pagbaybaybay ng wikang pinagsasalinan. Ayon kina Nida at Taber (1982) nakukuhang ganap ang naturalisasyon ng isang salin kung maintindihan ang kultural na konteksto ng wika at ang target na mambabasa kaya dapat tingnang maigi ang pinakamalapit na tumbas ng salita na may pagsasaalang-alang sa naturang mga aspekto. Sa isinagawang pagsasalin, nagkaroon ng adapsyon sa salitang "barrio" na sinunod ang pagbabaybay sa salita sa pamamagitan ng tuntuning "siyang bigkas, siyang baybay" kaya ito ay naging "baryo". Nakita sa pag-aaral ni Liban-Iringan (2005-2006) ang ganitong pagsasalin kung saan tuwiran at ganap na inililipat ang ilang mga salita sa SL tungo sa TL upang makatulong sa ikalilinaw ng mensahe.

Pagdaragdag - Ang pagdaragdag ay paglalagay ng ilang salitang wala sa orihinal na wika upang bigyang-diin o ipaliwanag ang isang salita o konsepto. Ang ganitong pamamaraan ay makikita sa naging salin ng kuwentong Agluom Manen dagiti Lansones ni T. Gabriel Tugade, kung saan ang salitang "nagmataak" ay tinumbasan ng "binuksan ko ang aking mga mata". Mapapansin na nadagdagan ng mga salita ang pagtutumbas sa SL tungo sa TL. Natuklasan din ang ganitong pamamaraan ng pagsasalin sa naging pag-aaral nina Fadero et al. (2017), kung saan tinangka nilang isalin ang maikling kuwento ni Angelo Rodriguez Lacuesta na Life Before X at nakita ang pagdaragdag ng mga salita sa ginawang salin.

Pagkakaltas - Ito ay ang pagtatanggal o pagbabawas ng/mga salita at mga pangungusap sapagkat hindi na mahalaga at/o may kahirapan itong tapatan batay sa pananaw ng tagasalin. Ang ganitong pamamaraan ay makikita sa pagsasalin sa kuwentong Agkitemda Iti Malem. Makikita mula sa siniping pahayag ang tuwirang pagkakaltas at hindi pagtutumbas sa mga salita sa SL gaya ng salitang "bassit" na kinaltas sa pagsasalin sa TL dahil kahit hindi ito matutumbasan ay mananatili pa rin ang kontekstuwal na kahulugan ng isinagawang salin. Ito ang naging prinsipyo ni Ancheta (2019) sa kaniyang pag-aaral sa pagsasalin niya ng nobelang One Hundred Years of Solitude ni Gabriel Garcia Marquez at nakita niya ang malaking tulong ito sa pagpapakinis ng saling isinagawa.

Functional na Pagtutumbas - Ayon kay Newmark (1988) ito ay pamamaraan ng pagsasalin kung saan dinedekulturalisa ang wika. Sa pagsusuri sa mga salin ng kuwento, nakita ang pamamaraang ito sa kuwentong Nalabaga ti pasayak kung saan tinumbasan ang salitang "talunasan" ng "itak". Tinumbasan ang kagamitan ng isa pang gamit na naaangkop at may kahawig sa kultura ng target na wika. Napansin din ito sa pag-aaral na pagtatangkang pagsasalin ni Liban-Iringan (2005-2006) ng Palavvun at Iringan kung saan ginamit niya ang pamamaraang ito sa mga pahayag na nararapat bigyang kahulugan na angkop sa mensahe ng linya ng akda.

Kuplet - Ito ay ang pagsasama ng dalawang pamamaraan ng pagsasalin. Kapansin-pansin ito sa mga isinalin at sinuring mga kuwento kaya lalo pang napabuti ang pagtutumbas at pagsasalin sa mga ito. Mapapansin ang pamamaraang pagkakaltas sa unang bahagi ng pahayag dahil sa hindi tinumbasan ang salitang "batog" ngunit hindi ito nakaapekto sa kontekstuwal na pagpapakahulugan sa pahayag. Ginamit din ang pamamaraang pagdaragdag ng panghalip na "niya" sa TL upang higit na maihayag nang maayos ang pagtutumbas sa SL.

Pag-aangat sa/ng orihinal na wika (dagdag mula sa mananaliksik) - Bahagi ng adhikain at pagsusulong ng mga wika sa bansa at intelektuwalisasyon ng wikang Filipino ang pagtukoy, pagsangguni, at paghango sa 
mga wikang katutubo para tumbasan ang mga salitang mahirap isalin. Bilang tugon sa kahingiang ito, ang mananaliksik ay hindi tuwirang nagsalin ng mga salita mula sa SL tungo sa TL kahit na ito ay may tumbas na salin. Gaya na lamang ng salitang "bit-ang" na maaaring isalin sa TL na "makipot na daanan" pero hindi ito tinumbasang tuwiran dahil sa kagustuhang i-angat ang wikang Ilokano at gamitin ang salitang natukoy bilang tumbas at mas maikling tawag sa bagay na natukoy.

\subsection{Katangian ng mga Naisaling Akda Batay sa Pagsusuri ng mga Eksperto}

\section{Talahanayan 2}

Katangian ng mga Naisaling Akda

\begin{tabular}{|c|c|}
\hline Katangian & Pahayag mula sa mga Eksperto \\
\hline Pagiging Matapat ng salin (Literal) & $\begin{array}{l}\text { Nagawang isalin ng mananaliksik ang mga piyesa sa } \\
\text { pinakamalapit na katumbas nito sa orihinal. }\end{array}$ \\
\hline Pagiging Malaya ng salin & $\begin{array}{l}\text { Napansin na malaya ang isinagawang pagsasalin sa ibang } \\
\text { mga akda ngunit hindi ito nakaapekto sa nais palitawing } \\
\text { pahayag mula sa simulaang wika tungo sa tunguhang wika. }\end{array}$ \\
\hline $\begin{array}{l}\text { Pagsasaalang-alang sa Kultura } \\
\text { ng orihinal na akda } \\
\text { Pagiging angkop ng mga salin }\end{array}$ & $\begin{array}{l}\text { Tama 'yong ginawa ng tagasalin na hindi na isinalin ang } \\
\text { mga katutubong salita dahil wala itong tumbas sa Filipino. } \\
\text { Angkop ang naging pagsasalin sa mga kuwentong bayan at } \\
\text { nakita ang pagsasaalang-alang sa ilang mga pamantayan ng } \\
\text { pagsasalin. }\end{array}$ \\
\hline
\end{tabular}

Batay sa isinagawang pagsusuri, napansin ng mga ebalweytor na may mga tinumbasang salita sa simulaang lengguwahe (SL) sa pinakamalapit na estruktura ng target na lengguwahe (TL) kaya naging matapat o literal ang naging pagsasalin ng mga ito. Hindi naman nakaapekto ito sa nais palitawing kahulugan sa target na lengguwahe. Napansin din ng mga ebalweytor ang pagiging malaya ng pagsasalin o walang pagsasaalang-alang sa estruktura o ng orihinal na wika at may mga salita mula sa SL ang hindi kayang tumbasan ng literal na salin sa TL, kayat nagkaroon ng pagsasalin nang malaya sa pamamagitan ng paggamit ng idyoma ngunit may pagsisigurado sa pagpapanatili ng kahulugan at diwa ng pangungusap. Natuklasan din sa isinagawang pagsasalin ang tuwirang paghango ng mga katutubo o kultural na salita sa SL dahil walang salita o terminong tutumbas sa TL. Nakita at nasuri din na angkop ang naging pagsasalin at pagtutumbas ng mga salita, pangungusap, at mga akda at isinaalang-alang ang mga konsepto at simulain sa pagsasalin.

\subsection{Kalinangan ng mga Ilokano na Napapaloob sa mga Kuwentong Bayan}

\section{Talahanayan 3}

Kalinangan ng mga Ilokano na Napapaloob sa mga Kuwentong bayan

\begin{tabular}{|c|c|c|}
\hline Tema & Ispesipikong Kalinangan & Halimbawa ng Pahayag \\
\hline $\begin{array}{l}\text { Kalinangan sa Hanapbuhay } \\
\text { ng mga Ilokano }\end{array}$ & Pagsasaka, Pangingisda, at Pangangaso & $\begin{array}{l}\text { Ipinukol ni Sencio ang kaniyang } \\
\text { mga mata sa hanay ng mga tanim } \\
\text { na mais na malapit ng } \\
\text { mamulaklak. Malalanta na ang } \\
\text { mga ito, bulalas niya. Dalawa o } \\
\text { tatlong araw pa ang hihintayin, } \\
\text { malalanta na ang mga dahon. }\end{array}$ \\
\hline $\begin{array}{l}\text { Kalinangan sa } \\
\text { Pamilyang Ilokano }\end{array}$ & $\begin{array}{l}\text { Pagmamahal ng/sa Magulang, } \\
\text { Pag-aalaga sa mga Maysakit }\end{array}$ & $\begin{array}{l}\text { Baka pumunta na naman si Isiang } \\
\text { sa klinika ni Doktor Cortes. Halos } \\
\text { araw-arawin ni Doktor Cortes ang } \\
\text { pagdalaw nito kaniya, noong hindi } \\
\text { pa gaanong Malala ang sakit ni } \\
\text { Basilio. Matagal ang naging } \\
\text { pag-uusap nina Doktor Cortes at } \\
\text { Isiang sa gilid kung kailangan na } \\
\text { bang turukan ng manggagamot } \\
\text { ang likod ni Basilio, dahil nasira } \\
\text { na ang kaniyang balikat. }\end{array}$ \\
\hline
\end{tabular}


Tipon, salin, at suri ng mga kuwentong bayang Iloko

Positibong Pag-uugali ng mga Ilokano

Negatibong Pag-uugali ng mga Ilokano
Pananampalataya sa Diyos, Pagtanaw ng Utang na Loob, Pagpapahalaga sa Edukasyon, Masipag at mapagmahal sa trabaho, Magpapakumbaba, Matiisin, at Mapagpatawad

Pang-aabuso sa kapangyarihan, Kapit o padrino system, Pagsusugal
May awa ang Diyos. Masaya ako sa piling mo. Hinigpitan niya ang pagyakap sa kaniyang asawa. Di ko alam ang aking gagawin... ang mangyayari sa buhay ko kung wala ka.

Binalasa ni Narding ang makapal na baraha. Pagkatapos, kinuha niya ang nasa ibaba at kaniya itong inilatag sa ibabaw ng mesa. Maghintay ka muna dahil naiihi na ako, pinigil siya ng kaniyang kaharap, si Conrado, sa kaniyang pamimigay.

Kalinangan sa Hanapbuhay ng mga Ilokano - Dahil sa topograpiya ng rehiyong kinabibilangan ng mga akda, marami sa mga mamamayan ang may trabahong may kinalaman sa pagsasaka, pangingisda, at iba pang may kinalaman sa bukid, bundok, at dagat. Mula sa pagsusuri ng mga kuwentong bayan, nakita ang pagsasaka, pangingisda, at pangangaso na kalinangan sa hanapbuhay ng mga Ilokano. Ang pagsasaka ang isa sa mga pangunahing ikinabubuhay ng mga Ilokano. Dahil sa ganitong pamumuhay at lokasyon ng nasabing rehiyon, malaki ang naging epekto nito sa mga manunulat ng panitikang Ilokano o Kurditan. Ginamit at ginagamit ng mga manunulat ang mga ito bilang lokasyon o ganapan ng kanilang naisulat at nailimbag na mga kuwento at iba pang uri ng panitikan.

Kalinangan ng Pamilyang Ilokano - Mahalaga sa mga Ilokano ang kanilang pamilya o mahal sa buhay. Mahalaga ang papel ng bawat isa sa loob ng isang tahanan, kinakailangang mahubog at malaman ng bawat isa ang tungkulin ng mga ito upang maging matiwasay ang isang pamilya. Ang pagmamahal sa/ng magulang ang isa sa mga nakita. Dakila ang pagmamahal ng magulang. Handa silang magsakripisyo at magtiis alang-alang sa kanilang mga mahal sa buhay lalong-lalo na sa kanilang mga anak. Nakita rin ang kabutihan ng mga Ilokano sa pag-aalaga sa maysakit. Makikita ang ganitong pagpapahalaga sa ipinamalas ng katauhan ni Isiang sa kuwentong Agkitemda Iti Malem. Mapapansin na sa kabila ng hirap ng buhay at sakit na tinatamasa ay hindi pa rin nawawala ang pagmamahal at pagmamalasakit sa isa't isa. Hindi nawawala ang ganitong pagpapahalaga ng mga Ilokano na sa kabila ng mga pasakit sa buhay ay nananatili silang matatag para sa mga kapamilyang patuloy na kumakapit sa kanila.

Positibong Pag-uugali ng mga Ilokano - Kailanma'y hindi nawawala sa pag-uugali ng mga Ilokano ang mga positibong pagpapahalaga na siyang lalong nagpapadakila sa katauhan ng mga mamamayan. Batay sa naging pagsusuri ng mga kuwentong bayan, natuklasan ang ilang mga positibong kalinangang pagpapahalaga na tunay na taglay ng mga Ilokano gaya ng pagtanaw ng utang na loob, pagpapahalaga sa edukasyon, pagiging mapamahiin, pagiging masipag at mapagmahal sa trabaho, mapagpakumbaba, mapagpatawad, at matiisin. Tunay na ang mga pag-uugaling ito ay pagkakakilanlan na ng mga Ilokano dahil karugtong na ito ng kanilang kalinangan o kulturang kinagisnan.

Negatibong Pag-uugali ng mga Ilokano - Sa kabila ng mga positibong pagpapahalagang taglay ng mga Ilokano ay kakikitaan din ng mga negatibong pag-uugali. Mula sa naging pagsusuri ng mga kuwentong bayan, nakita ang ilang negatibong pagpapahalaga ng mga Ilokano gaya ng pang-aabuso sa kapangyarihan, sistema ng kapit o padrino, at pagsusugal. Maraming mga sitwasyon sa mga akdang sinuri ang kakikitaan ng di kagandahang asal na taglay ng mga tauhan na bahagi rin ng kalinangan ng mga Ilokano.

\section{Kongklusyon at Rekomendasyon}

Nabuo ang sumusunod na kongklusyon pagkatapos masuri at mabigyang-kahulugan ang mga datos na nakalap: ang mga pamamaraan ni Newmark (1988) ay angkop gamitin sa pagsasalin ng mga kuwentong bayan; ang pag-aangat ng ilang mga termino sa SL sa pagsasalin ay makatutulong sa intelektuwalisasyon ng isang wika; 
ang mga salin lalo na sa kuwentong bayan ay dapat angkop at kontekstuwalisado; at ang mga kuwentong bayan ay kinapapalooban ng mga kalinangan ng mga pangkat na nagmamay-ari nito. Batay sa mga natuklasan at kongklusyong nabanggit, iminumungkahi na gamitin ang mga pamamaraan ni Newmark (1988) sa pagsasalin ng mga akdang tuluyan tulad ng kuwentong bayan at ipasuri ang mga ginawang pagsasalin para matiyak ang kaangkupan nito; gamitin ang konseptong pagsasalin na iminungkahi ng mananaliksik na pag-aangat sa orihinal na wika; tangkilin at basahin ang mga panitikang lokal dahil maraming mga pagpapahalaga ang nakalapat sa mga ito na maaaring magamit bilang lunsaran sa pagtuturo at pagkatuto; hinihikayat ang mga tagapagtaguyod ng karunungan-- ang mga guro na bumuo ng mga kagamitang panturo na nakaangkla sa mga akdang pampanitikang lokalisado; patuloy na tipunin, isalin, at dalumatin ang mga akdang pampanitikan sa bansa lalong-lalo na ang mga unti-unti nang nakakalimutan para mapreserba at mapayabong na rin ang wika at panitikan sa bansa; at sa mga mananaliksik, magsisilbing gabay nila ang pananaliksik na ito upang ipagpatuloy ang pag-aaral sa pagsasalin at pagsusuri sa mga akdang pampanitikang lokalisado para lalo pang mapaangat ang kalagayan ng panitikan sa bansa.

\section{References}

Almario, V. S. (2015). Introduksiyon sa Pagsasalin. Komisyon sa Wikang Filipino.

Ancheta, J. (2019). Tawid-Kultural na Pagsasalin ng Nobelang One Hundred Years of Solitude ni Gabriel Marquez: Mga Estratehiya at Suliranin. Dalumat E-Journal Isyu 2019, Tomo 5, Blg. 1.

Catipay, T. A. (2019). Antolohiya ng mga Sugbu-anun'g Kwentong Bayan: Pagbabalik-tanaw sa buhay kulturang Cebuano. Asia Pacific Journal of Multidisciplinary Research,7(1).

Du, N. O. (2014). Pagdalumat Sa Mga Sinaunang Di- Materyal Na Kultura Ng Mga B'laan Sa Brgy. Pisan, Kabacan, Cotabato Mula Sa Kanilang Kwentong Bayan. Asia Pacific Journal of Multidisciplinary Research,2(6). http://www.apjmr.com/wp-content/uploads/2014/12/APJMR-2014-2-165a-Pagdalumat-Sa-Mga-Sinaun ang-Di-Materyal-Na-Kultura.pdf

Fadero, J., K. Garzon, K. Matibag, \& Suarez, K. (2017). Pagsasa-Filipino ng Maikling Kuwento ni Angelo Rodriguez Lacuesta Life Before X. Batangas State University.

Hidalgo, J. S. P. (1968). Napili a sarita para dagiti Ilokano.

Hsiu-Fang, H. (2005). Three approaches to qualitative content analysis. Qualitative Health Research. https://doi.org/10.1177/1049732305276687

Jamilosa-Silapan, O. (1993). Panagiyulog: Tungo sa Pagbubuo ng Isang Teory sa Pagsasalin (Maikling Kuwentong Iloko sa Filipino. PhD Fil. UP Diliman.

Kondracki, N., \& Wellman, N. (2002). Content analysis: review of methods and their applications in nutrition education. PubMed.gov, 34(4).

Liban-Iringan, E. (2005-2006). "Pamalittac": Pagsasalin ng mga "Palvvun" at "Unoni” ng Ibanag at Itawes bilang Bahagi ng Pambansang Panitikan. PhD Fil. UP Diliman.

Newmark, P. (1988). Pragmatic translation and literalism. TTR: Traduction, Terminologie, Rédaction, l(2), 133. https://doi.org/10.7202/037027ar

Potter, W. J., \& Levine-Donnerstein, D. (1999). Rethinking validity and reliability in content analysis. Journal of Applied Communication Research, 27(3), 258-284. https://doi.org/10.1080/00909889909365539

Santos, T. U. (2008, November 17). Pakikibaka sa makabayang panitikan. The Varsitarian.

Whittlessey, H. (2012). A typology of derivatives: Translation, transposition. Adaptation, 16(12).

Young, J. (1994). Philippine myths and legends. Metro Manila: MG Reprographics. 